\title{
Virtual long-period gratings
}

\author{
César Jáuregui and José Miguel López-Higuera \\ University of Cantabria, Grupo de Ingeniería Fotónica, Departamento Tecnología Electrónica e Ingeniería Sistemas y Automática, \\ Avenida Los Castros s/n, c.p. 39005 Santander, Spain \\ Received July 13, 2004
}

\begin{abstract}
A theoretical prediction of a new fiber grating structure is presented. This new device is achieved when a high-birefringence fiber is heavily twisted. It behaves like a long-period grating, but its spectral response can be controlled with the input state of polarization of the light. Since there is no permanent index change, but the index change is instead a product of the polarization evolution along the fiber, this device is called a virtual long-period grating. (c) 2005 Optical Society of America
\end{abstract}

OCIS codes: $\quad 060.2400,060.2340$.

Conventional fiber Bragg gratings (FBGs) and longperiod gratings (LPGs) have been important devices for both the sensing and the telecommunication fields. They have served as the basis for developing sensors for the most diverse parameters (strain, temperature, pressure, chemical compounds, etc. $)^{1,2}$ and devices for telecommunication networks ${ }^{3}$ (dispersion compensation devices, filters, etc.). However, this technology has finally reached a certain degree of maturity, and research interests are beginning to abandon conventional gratings. As a result, two main new research paths have been opened in the grating field. One of them concerns more exotic grating-based structures, such as those called FBGs. ${ }^{4}$ The other tries to overcome one of the main problems of conventional grating technology: the lack of flexibility of already-written gratings. Since FBGs and LPGs appear after a permanent UV-induced index change is created in the core of a fiber, there is little room for modifying their spectral responses after they have been written. However, it would be interesting to have a controllable gratinglike device. Among its most obvious applications would be tunable filters or new sensors. For this reason, some research effort has recently been devoted to obtaining controllable (electronically or with light) devices that behave as gratings. Within this framework a tunable LPG written in Er-doped fiber $^{5}$ or a completely reconfigurable filter ${ }^{6}$ are recent achievements. In addition, we recently introduced a novel structure: the virtual long-period grating (VLPG). ${ }^{7}$ The model presented in Ref. 7, together with its results, is corrected and expanded here.

A VLPG is a structure that behaves essentially as a LPG but whose spectral characteristics can be controlled with the input state of polarization (SoP) of the light. This structure is achieved when heavily twisting a high-birefringence (HiBi) fiber. Under these circumstances the SoP of the light is continuously modified. This SoP evolution gives rise to a periodic change in the effective index of refraction of the propagating light. The shape of this effectiveindex change depends on the initial SoP of the light. Therefore, since there is no permanent index change in the fiber, the already-mentioned control of the VLPG's characteristics is possible. In the following we explain how this effective-index change takes place.
In a $\mathrm{HiBi}$ fiber there are two orthogonal linear SoPs, known as the eigenmodes of the fiber, that travel unperturbed along it. However, these two SoPs travel at different speeds; i.e., they experience different effective indices $\left(n_{1}\right.$ and $\left.n_{2}\right)$. This implies that in a $\mathrm{HiBi}$ fiber the effective index experienced by the propagating light is polarization dependent. This property is the basis of the VLPG.

It is widely known that any $\mathrm{SoP}$ can be decomposed into a combination of the two eigenmodes. Thus the effective index experienced by this light will depend on its decomposition into the eigenmodes. From this point of view an exchange of energy between the two eigenmode components of a $\mathrm{SoP}$ is mandatory for an effective-index change to happen. However, this will never occur in a plain HiBi fiber. This exchange of energy is possible only by means of a relative rotation of the SoP with respect to the eigenaxis of the fiber.

As roughly illustrated in Fig. 1, the latter can be achieved by twisting a HiBi fiber [in the case of Fig. 1(a) an elliptical-core fiber]. In such an environment the SoP does not follow the eigenaxis; i.e., if an eigenmode is introduced at the beginning of the

(a)

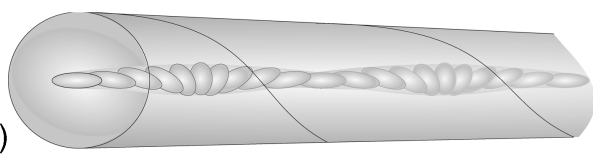

(b)

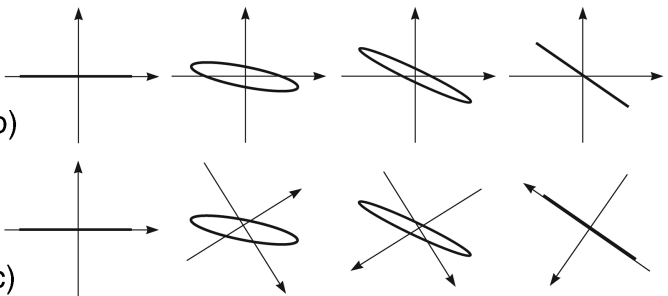

(c)

(d)

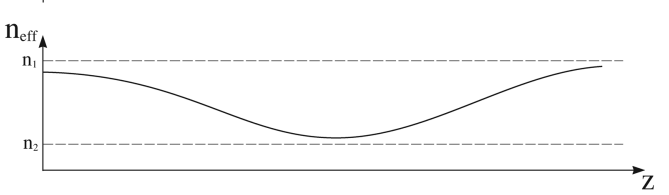

Fig. 1. (a) Heavily twisted HiBi fiber that generates the VLPG. (b) SoP evolution with respect to a static reference system. (c) SoP evolution as seen from a reference system attached to the eigenaxis of the fiber. (d) SoP-dependent effective-index change seen by the propagating light. 
twisted fiber, it will not rotate following its eigenaxis. This is shown schematically in Fig. 1(b), in which the SoP evolution of the light with respect to a static reference system is tracked along the fiber. The light was originally polarized with one of the eigenmodes, but, as has already been said, it does not rotate as it propagates through the twisted fiber. On the contrary, it changes its ellipticity due to the effect of the linear birefringence and rotates a little due to the torsion-induced circular birefringence. This loose relationship between the $\mathrm{SoP}$ and the eigenaxis eases the appearance of the effective-index change. This is because, as shown in Fig. 1(c), there is a relative rotation between the eigenaxis and the SoP.

The effective index of any particular SoP can be estimated by decomposing it into the eigenmodes and by taking a weighted average of $n_{1}$ and $n_{2}$. To do so, the appropriate reference system is that shown in Fig. 1(c), i.e., that attached to the eigenaxis of the fiber. Following the procedure described above, effective-index profiles such as that shown in Fig. 1(d) can be obtained. These profiles, which are periodic, correspond go gratinglike structures but depend on the initial SoP of the light. They are not permanent; they are experienced by the light only as it propagates through the fiber. In that sense they are virtual, which is why these structures have been called virtual gratings.

In summary, there are two main factors that contribute to the appearance of a virtual grating: the linear birefringence of the fiber and the relative rotation, induced by the twist, between the SoP and the eigenaxis. However, there is yet another factor that plays an important role in configuring the effective-index change profile: the torsion-induced circular birefringence. However, because this parameter is usually not very high, its effect is mainly focused on slightly modifying the period of the effectiveindex profile, i.e., the period of the virtual grating.

To provide mathematical support for this discussion, the general Jones matrix for an optical fiber of evenly distributed retardations can be used. ${ }^{8}$ First of all, let us define a parameter that gives an idea of the total amount of polarization change $\lambda_{T}=\left[\left(\delta_{l} / 2\right)^{2}+(\tau z+\right.$ $\left.\left.\delta_{c} / 2\right)^{2}\right]^{1 / 2}$. Thus, with this parameter, the SoP of the light referred to the eigenaxis at any point of the fiber can be obtained by use of the following matrix:

$$
T=\left[\begin{array}{cc}
P & -Q^{*} \\
Q & P^{*}
\end{array}\right],
$$

where

$$
\begin{aligned}
& P=\cos \lambda_{T}-j \frac{\delta_{l}}{2} \frac{\sin \lambda_{T}}{\lambda_{T}}, \\
& Q=\left(\tau z+\frac{\delta_{c}}{2}\right) \frac{\sin \lambda_{T}}{\lambda_{T}} z .
\end{aligned}
$$

Here, $\delta_{l}=2 \pi \Delta n z / \lambda$ is the cumulative linear birefringence, with $\Delta n$ being the index difference between the two eigenmodes; $\lambda$ is the wavelength of the light; and $z$ is the position along the fiber. $\delta_{c}=-2 g \tau z$ is the cumulative circular birefringence, where $g$ is a factor whose value ranges from 0.065 to 0.08 for most fibers, $\tau$ is the twist ratio (in radians per unit length), and $z$ is the length of the fiber to the point at which the SoP is going to be calculated. Thus, by application of this matrix to the initial SoP and for different points along the fiber, a SoP evolution can be obtained. Because this $\mathrm{SoP}$ is always referred to the eigenaxis by means of Eq. (1), it can be directly decomposed into its components. Then, taking a weighted average of the effective indices of the eigenmodes $\left(n_{1}\right.$ and $\left.n_{2}\right)$ allows us to obtain the effective index of the calculated SoP. Therefore repeating this procedure at different points along the fiber allows us to obtain an effectiveindex profile. Now the spectral characteristics of the structure described by this index profile can be calculated with coupled-mode theory. ${ }^{9}$

If the eigenvectors of Eq. (1) are calculated, the SoPs that travel unperturbed along the twisted fiber will be known. These SoPs are important, since they are those for which no index change is seen and, therefore, no virtual grating exists. These eigenvectors $\left(\left[A_{v}, B_{v}\right]\right)$ are given by

$$
A_{v}=\frac{ \pm j\left(\tau z+\delta_{c} / 2\right)}{\lambda_{T} \pm \delta_{l} / 2} B_{v} .
$$

By making a few calculations, we can verify that these eigenvectors are independent of both the grating length and the position along the grating $(z)$. They depend only on the birefringence characteristics of the fiber, on the twist rate, and on the wavelength. This result corroborates that these eigenvectors represent true eigenmodes of the VLPG that travel unperturbed along the twisted fiber.

By using this model, we simulated several twisted HiBi fibers and found that, to obtain resonances in the $1300-1600-\mathrm{nm}$ range, twist ratios of approximately 1000 turns $/ \mathrm{m}$ are needed. This is far beyond what we were able to achieve in the laboratory (a maximum of approximately 200 turns $/ \mathrm{m}$ ). Therefore only simulation results are presented in this Letter. From these simulations and for that twist ratio, gratings with periods of several hundred micrometers were obtained. This explains why these virtual gratings are defined as long period.

The results presented and discussed in the following were obtained from spectra calculated with coupledmode theory by use of the index profiles obtained with the model already described. This task was carried out with the commercial software IFO Gratings (Optiwave). The simulations were performed by always assuming a constant twist ratio of 1330 turns $/ \mathrm{m}$ and the same host fiber (a HiBi fiber of $\Delta n=6 \times 10^{-4}, 1.4455$ average core index, 1.4452 cladding index, $4.5-\mu \mathrm{m}$ core radius, $62.5-\mu \mathrm{m}$ cladding radius, $20-\mathrm{cm}$ length, and surrounded by air). The cladding modes involved in the simulations were those of a standard fiber, which is a good approximation for elliptical-core fibers.

Thus Fig. 2 shows the extreme changes that the spectrum of a single VLPG can suffer simply from modification of the input SoP of the light. There are changes in the attenuation, the central wavelength, the spectral width, and even the spectral shape. These spectra are strongly length dependent and can therefore be altered simply by use of a longer VLPG (these 


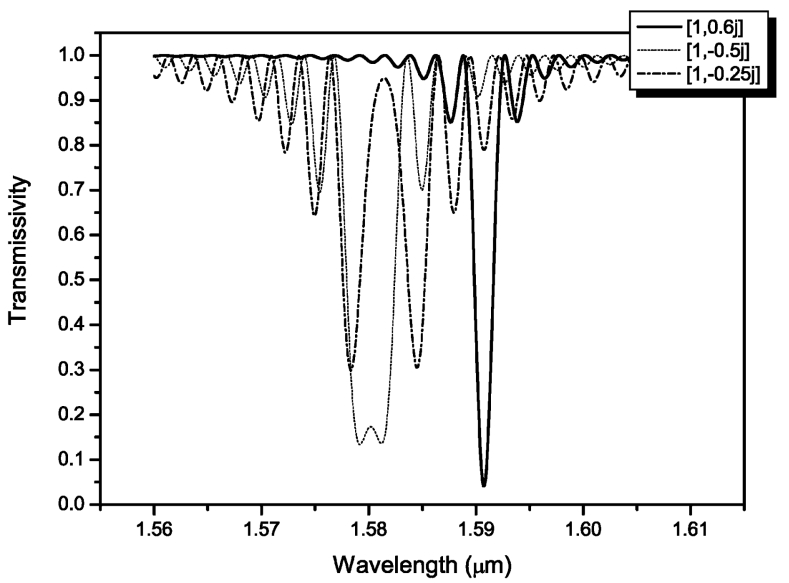

Fig. 2. Spectra of a 1330-turn/m VLPG for three different input polarizations: $[1,0.6 j]$ (solid curve), $[1,-0.5 j]$ (dotted curve), $[1,-0.25 j]$ (dashed-dotted curve).

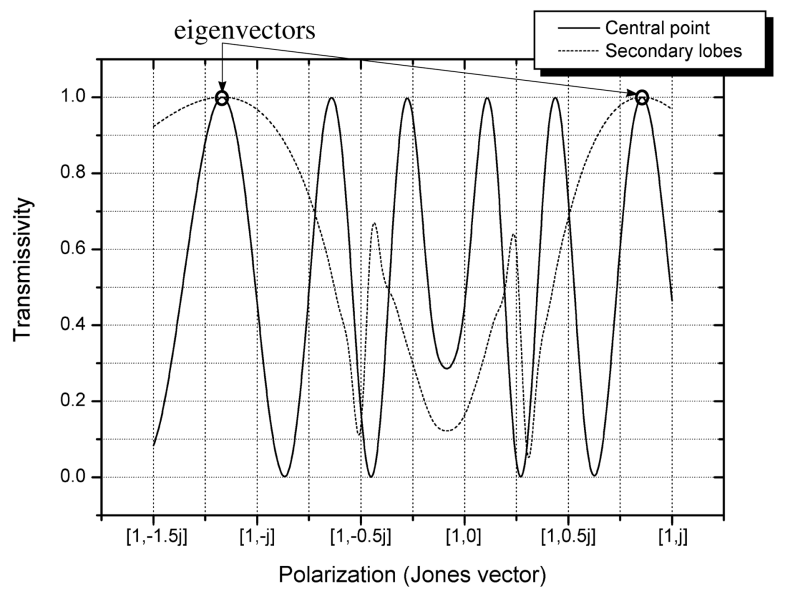

Fig. 3. Transmissivity change of a VLPG for different initial SoPs. The transmissivity of the middle point of the spectrum (solid curve) and the secondary lobes (dotted curve) are plotted. The SoPs at which the VLPG vanishes are marked.

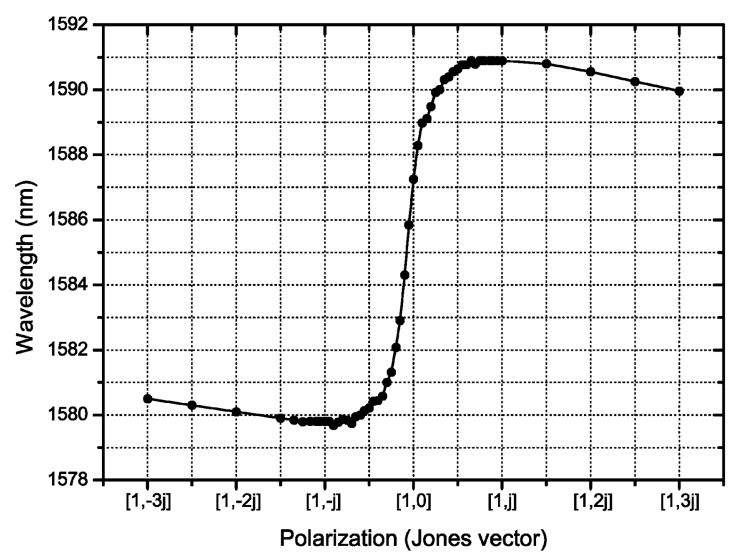

Fig. 4. Central wavelength shift for different initial circular SoPs.

structures can be easily made meters long). To quantitatively evaluate this spectral change, Figs. 3 and 4 are presented. In Fig. 3 the attenuation of the central point of the spectrum (solid curve) and the main secondary lobes is plotted against initial circular SoPs. From this figure the extreme spectral distortion already mentioned can be induced. There are SoPs at which the sidelobes present high transmissivity values and the central point attenuation is very high (i.e., more than 90\%; spectrum such as that shown in Fig. 1 for $[1,0.6 j])$ and others at which the sidelobes exhibit transmittance values lower than 50\% (to the extent that they can be considered two main lobes). There are also two points [given by Eq. (2)] marked in the graph at which the VLPG vanishes. The SoP sweep for linear polarizations yields a much softer variation than the one shown in Fig. 3. This means that for linear polarizations the spectrum shifts its central wavelength without suffering much distortion.

Figure 4 shows the central wavelength shift for the same circular SoP sweep of Fig. 3. It can be seen that a wavelength change of almost $15 \mathrm{~nm}$ is achievable. Besides, the wavelength shift is abrupt around $[1,0]$, a fact that can be used for making sensors. With linear polarizations the total wavelength shift is approximately $4 \mathrm{~nm}$ and less steep.

In summary, a new structure called a virtual long-period grating has been, for the first time to our knowledge, presented and theoretically predicted. It behaves like a normal LPG except for the fact that its spectral signature can be modified by means of the input SoP of the light. This device can find application in both the sensor and the telecommunication fields. We are currently working on an experimental demonstration of the VLPG.

This work was cosupported by the Spanish Comisión Interministerial de Ciencia y Tecnología TIC-2001-0877-C02-01 and the TIC-2001-4503-E projects. C. Jáuregui's e-mail address is jauregui@ teisa.unican.es.

\section{References}

1. J. M. López-Higuera, Ed., Handbook of Optical Fiber Sensing Technology (Wiley, New York, 2002).

2. J. Dakin and B. Culshaw, eds., Optical Fiber Sensors: Applications, Analysis, and Future Trends (Artech House, Norwood, Mass., 1997).

3. A. M. Vengsarkar, P. J. Lemaire, J. B. Judkins, V. Bathia, T. Erdogan, and J. E. Sipe, J. Lightwave Technol. 11, 1513 (1993).

4. M. J. Holmes, R. Kashyap, and R. Wyatt, IEEE J. Sel. Top. Quantum Electron. 5, 1353 (1999).

5. M. A. Quintela, D. A. González, F. J. Madruga, M. Lómer, and J. M. López-Higuera, Proc. SPIE 5502, 431 (2004).

6. J. J. Russel, M. A. Davis, J. Sirkis, A. D. Kersey, and H. Lara, Proceedings of the 16th Optical Fiber Sensors Conference (Institute of Electronics, Information and Communication Engineers, Tokyo, Japan, 2003), pp. $538-541$.

7. C. Jáuregui, D. A. González, M. A. Quintela, P. Marqués, A. Cobo, and J. M. López-Higuera, Proc. SPIE 5502, 447 (2004).

8. C. Tsao, Optical Fiber Waveguide Analysis (Oxford U. Press, London, 1992).

9. W. P. Huang, J. Opt. Soc. Am. A 11, 963 (1994). 